\title{
AGENTS WITHIN A DEVELOPMENTAL COMPLEX ADAPTIVE SYSTEM: INTRAUTERINE MALE HORMONES INFLUENCE HUMAN TOOTH SIZE AND SHAPE
}

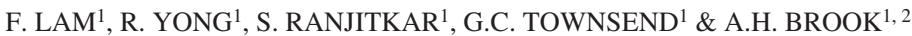 \\ ${ }^{1}$ School of Dentistry, University of Adelaide, Australia. \\ ${ }^{2}$ Institute of Dentistry, Queen Mary University of London, England.
}

\begin{abstract}
Dental development is a complex adaptive system (CAS) in which genetic, epigenetic and environmental factors interact during different developmental stages to influence final tooth size and shape. From these complex interactions, a self-regulating and self-organising system emerges with spatiotemporal, multidimensional, multilevel and multifactorial components. The aim of this study was to quantify the influence of intrauterine male hormones on the mesiodistal dimension of permanent teeth by studying females from opposite-sex (OS) and same-sex (SS) twin pairs to explore dental development as a CAS. A custom-designed 2D image analysis system was used to measure the mesiodistal dimension from the occlusal view in each permanent tooth of 30 dizygotic OS and 30 dizygotic SS female twin pairs. Linear mixed models were used to quantify the differences in tooth size and shape. The mean mesiodistal tooth dimension was greater in the OS cohort for the majority of the permanent dentition. These findings reflected the complex interactions between genetic, epigenetic and environmental factors and their outcomes during development. The results indicated the transmission of the male hormones to the female in utero influencing her endocrine system, leading to epigenetic changes and so affecting gene expression. This demonstrated prenatal sex hormones as an environmental agent influencing multiple interactions within a developmental CAS.
\end{abstract}

Keywords: complex adaptive systems, dental development, epigenetic, genetic, phenomics, prenatal sex hormones, sexual dimorphism, Y chromosome.

\section{INTRODUCTION}

\subsection{The human dentition as a complex adaptive system}

Dental development is a complex adaptive system (CAS) emerging from interactions between genetic, epigenetic and environmental factors during the initiation, morphogenesis, differentiation, calcification and eruption of teeth [1,2]. Understanding the spatiotemporal, multidimensional, multilevel and multifactorial components of dental development has the potential to improve the knowledge of the role of CASs in craniofacial and general developments. Interactions between genetic, epigenetic and environmental factors on dental development can influence sexual dimorphism observed in the dentition, such as the final tooth size and shape [3].

\subsection{Sexual dimorphism within the dentition}

The phenotypic difference between males and females within the same species has been found in many human characteristics such as bone and teeth. Sexual dimorphism has been identified within 
different tooth dimensions of the primary and permanent dentitions and is influenced by several factors, including the Y chromosome and intrauterine male hormones [3].

\subsubsection{Influence of Y chromosome}

The Y chromosome is present in males, and it contains the SRY gene, which is influential in the testicular development and testosterone release [4]. The influences of the Y chromosome and genes on these characteristics require further investigation.

\subsubsection{Twin testosterone transfer hypothesis}

Testosterone release in utero can transfer from a male twin to the female co-twin, causing more masculine traits in the female [5]. By measuring a phenotype that develops at the time of the testosterone surges in utero in females from different twin types, it is possible to investigate the influence of intrauterine testosterone on the development of that phenotype. In addition, the intrauterine testosterone may have a priming effect, possibly with an epigenetic change that will influence tooth development later on.

\subsubsection{Epigenetics}

Epigenetic mechanisms alter the production of different cell types, forming different tissues and performing different functions, whilst still possessing the same DNA sequence [6-10]. Epigenetics involves "the addition or removal of methyl groups to DNA or the attachment of acetyl groups of histones" [11]. MicroRNAs are also epigenetically regulated to control a variety of biological functions in plants and animals [11].

\subsection{Dental phenomics}

Dental phenotypes such as tooth size and shape can be analysed using state-of-the-art, enhanced dental phenomic techniques such as two-dimensional (2D) and three-dimensional (3D) image analysis systems. The outcomes of such analyses can help to elucidate the developmental factors in health and disease [12]. A clear understanding of the various dental developmental variations and diseases can assist operators in forming a patient-centred diagnosis and treatment plan.

\section{AIMS}

The aim of this study was to quantify the influence of intrauterine male hormones on the mesiodistal dimension of permanent teeth between females from the opposite-sex (OS) and the same-sex (SS) twin pairs to explore dental development as a CAS.

\section{MATERIALS AND METHODS}

Measurements were obtained from all teeth present in the permanent dentition of the 30 female OS and 30 female SS twins, excluding the second and third molars as they were not fully formed. The dental casts were placed on a platform under standardised illumination as shown in Fig. 1. Each tooth was imaged from the occlusal view using a digital camera (Canon EOS 50D digital SLR camera, Cannon, Australia) with a resolution of 15.1 megapixels and image array of 4,752 × 3,168 pixels. The camera was positioned horizontally above the dental casts on an adjustable rod. Illumination involved four multidirectional spotlights surrounding the cast (Fig. 1). The camera was connected to a personal computer, which utilised imaging software. 


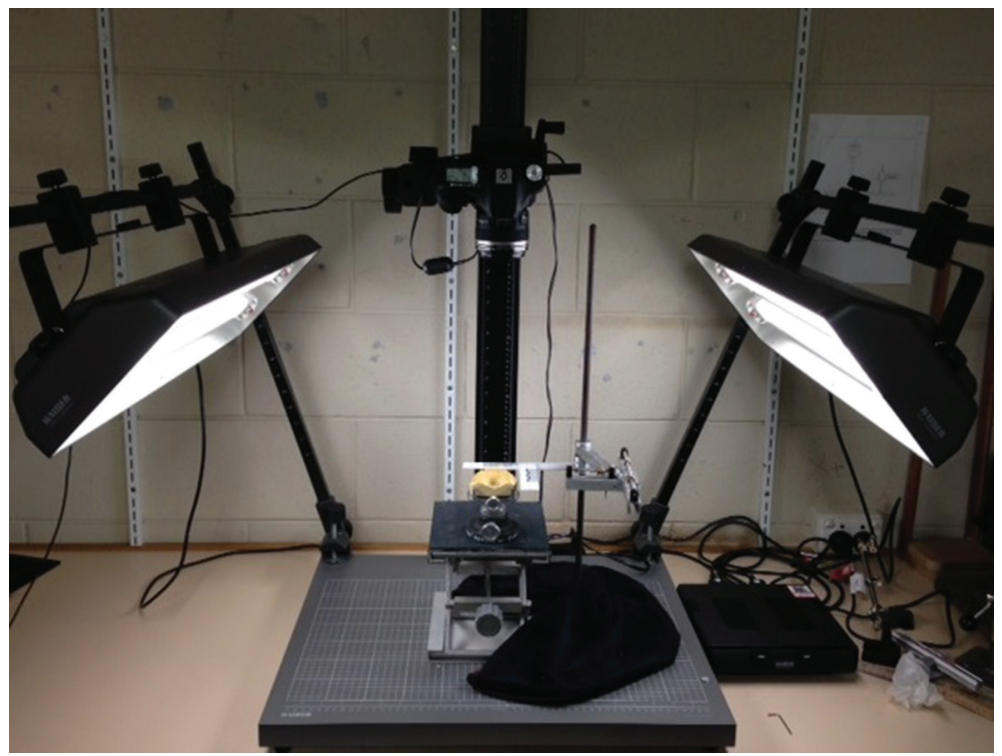

Figure 1: 2D image analysis system at the University of Adelaide Dental School.

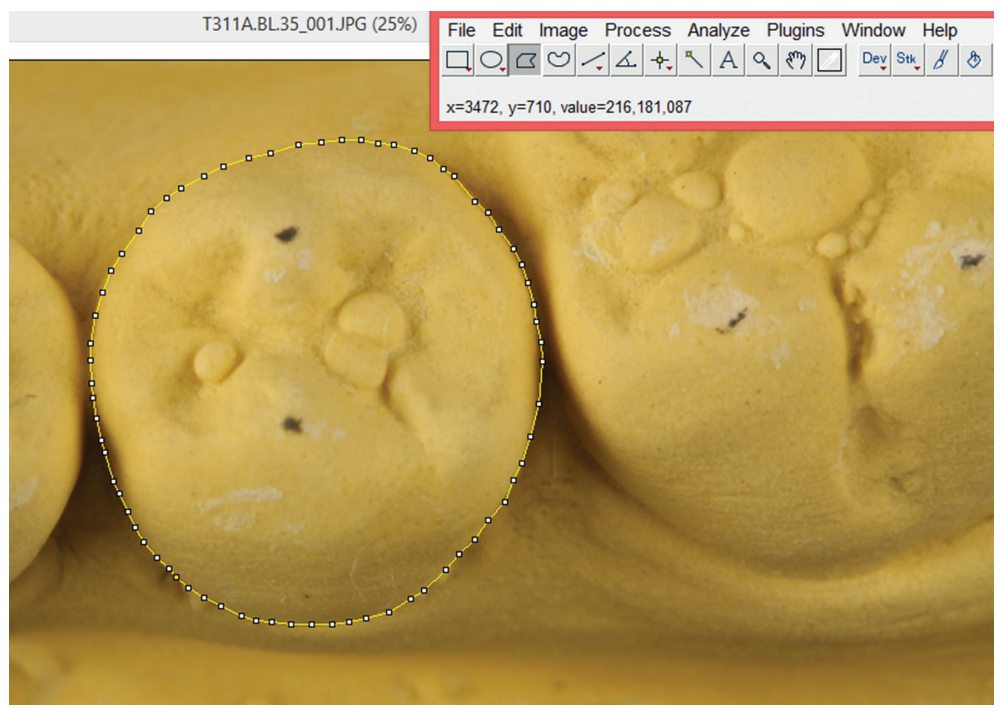

Figure 2: Mesiodistal (MD) width (blue line) measured on a permanent mandibular second premolar from the occlusal view.

The mesiodistal width represented the maximum distance between mesial and distal proximal surfaces of the tooth crown and was calculated from the occlusal/incisal (MD) view (Fig. 2). For each individual tooth and tooth types (incisors, canines, premolars and molars) in the mandibular and maxillary arches, descriptive statistics such as mean values and standard deviations were calculated for each zygosity. Linear mixed models were used to compare the variables for OS and SS females for individual teeth and the tooth types. 
Table 1: Comparison of mesiodistal widths (in millimetres) from occlusal view (MD) for every tooth between females from the OS and the SS cohorts.

\begin{tabular}{|c|c|c|c|c|c|c|}
\hline \multirow[b]{2}{*}{ MD } & & \multirow[b]{2}{*}{$\mathrm{p}(\mathrm{F})$} & \multicolumn{2}{|c|}{ OS Females $(n=30)$} & \multicolumn{2}{|c|}{ SS Females $(n=30)$} \\
\hline & & & Mean (mm) & $\mathrm{SD}(\mathrm{mm})$ & Mean $(\mathrm{mm})$ & $\mathrm{SD}(\mathrm{mm})$ \\
\hline \multicolumn{7}{|c|}{ Maxillary } \\
\hline \multirow{6}{*}{ Right } & I1 & 0.967 & 8.480 & 0.519 & 8.474 & 0.519 \\
\hline & $\mathrm{I} 2$ & 0.901 & 6.556 & 0.738 & 6.581 & 0.811 \\
\hline & $\mathrm{C}$ & 0.591 & 7.736 & 0.468 & 7.670 & 0.479 \\
\hline & $\mathrm{P} 1$ & 0.202 & 7.161 & 0.471 & 7.000 & 0.504 \\
\hline & $\mathrm{P} 2$ & 0.029 & 7.017 & 0.481 & 6.734 & 0.520 \\
\hline & M1 & 0.079 & 10.948 & 0.578 & 10.686 & 0.578 \\
\hline \multirow{6}{*}{ Left } & I1 & 0.755 & 8.478 & 0.518 & 8.520 & 0.518 \\
\hline & $\mathrm{I} 2$ & 0.837 & 6.644 & 0.586 & 6.611 & 0.636 \\
\hline & $\mathrm{C}$ & 0.67 & 7.574 & 0.490 & 7.519 & 0.512 \\
\hline & P1 & 0.121 & 7.158 & 0.437 & 6.979 & 0.455 \\
\hline & $\mathrm{P} 2$ & 0.209 & 6.989 & 0.448 & 6.837 & 0.483 \\
\hline & M1 & 0.577 & 10.729 & 0.548 & 10.650 & 0.548 \\
\hline \multicolumn{7}{|c|}{ Mandibular } \\
\hline \multirow{6}{*}{ Left } & I1 & 0.805 & 5.377 & 0.406 & 5.351 & 0.411 \\
\hline & $\mathrm{I} 2$ & 0.489 & 5.924 & 0.402 & 5.852 & 0.402 \\
\hline & $\mathrm{C}$ & 0.14 & 6.609 & 0.419 & 6.447 & 0.428 \\
\hline & $\mathrm{P} 1$ & 0.087 & 7.254 & 0.482 & 7.040 & 0.482 \\
\hline & $\mathrm{P} 2$ & 0.047 & 7.416 & 0.502 & 7.157 & 0.509 \\
\hline & M1 & 0.157 & 11.105 & 0.561 & 10.901 & 0.555 \\
\hline \multirow{6}{*}{ Right } & I1 & 0.813 & 5.445 & 0.407 & 5.421 & 0.407 \\
\hline & $\mathrm{I} 2$ & 0.972 & 5.978 & 0.411 & 5.974 & 0.414 \\
\hline & $\mathrm{C}$ & 0.295 & 6.646 & 0.429 & 6.528 & 0.440 \\
\hline & $\mathrm{P} 1$ & 0.133 & 7.173 & 0.505 & 6.976 & 0.511 \\
\hline & $\mathrm{P} 2$ & 0.053 & 7.470 & 0.525 & 7.206 & 0.532 \\
\hline & M1 & 0.076 & 11.079 & 0.588 & 10.812 & 0.582 \\
\hline
\end{tabular}

$\mathrm{I} 1$ = permanent central incisor; $\mathrm{I} 2$ = permanent lateral incisor; $\mathrm{C}=$ permanent canine;

$\mathrm{P} 1$ = permanent first premolar; $\mathrm{P} 2$ = permanent second premolar; $\mathrm{M} 1$ = permanent first molar; $\mathrm{n}=$ sample size $;$ mean = mean values; $\mathrm{SD}=$ standard deviation; $(\mathrm{p}<0.05)$; shading denotes direction of difference regardless of level of significance (bold) (red, OS $>$ SS; green, OS $<$ SS).

\section{RESULTS}

The mean values of MD widths showed a greater trend in the OS females compared with SS females for all teeth, except the maxillary right lateral incisors and left central incisors. The OS females 


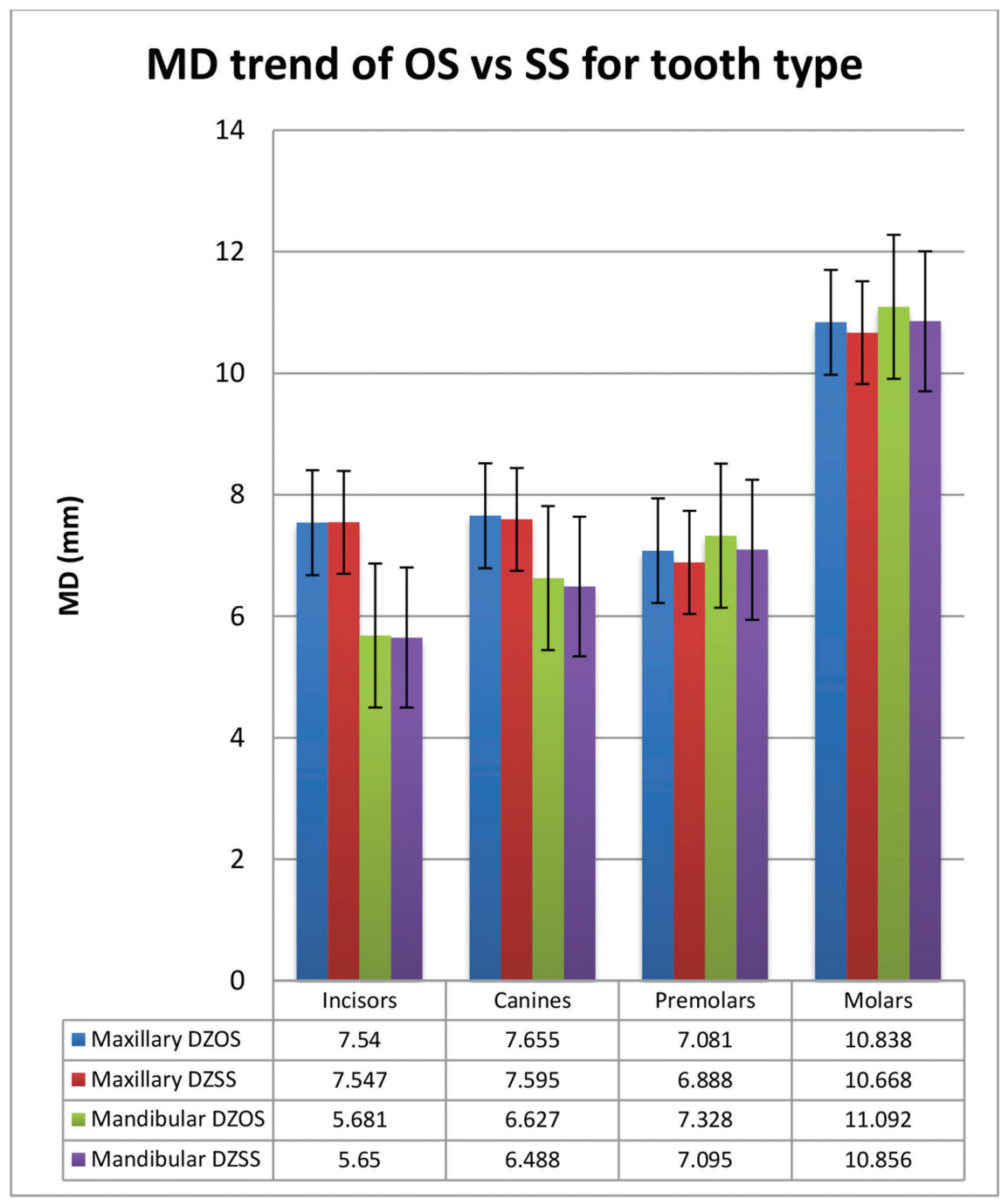

Figure 3: The mesiodistal (MD) widths of OS Females vs. SS Females for tooth type.

Blue = maxillary OS females, red = maxillary SS females; blue vs red for maxillary arch; green = mandibular OS females, purple $=$ mandibular SS females; green vs. purple for mandibular arch. Error bars represent standard errors.

showed significantly greater MD widths for the right second maxillary premolars and left mandibular second premolars in comparison to SS females $(\mathrm{p}<0.05)$ (Table 1).

The mean MD widths were significantly greater in the OS cohort than the SS cohort for maxillary and mandibular premolars when the teeth were grouped into four tooth types, excluding the maxillary incisors that displayed a difference of $0.007 \mathrm{~mm}(\mathrm{p}<0.05)$ (Fig. 3). The mandibular premolars and molars showed the greatest difference between OS cohort and SS cohort for the MD widths (Fig. 3). 


\section{DISCUSSION}

Using a twin model, this study further investigated the possible influence of prenatal sex hormones and the Y chromosome on tooth size and shape. Utilisation of tooth measurement data contributed to the understanding of the interactions of the genetic, epigenetic and environmental factors on tooth development. The tooth size and shape data made important contributions to the TTT hypothesis with regard to developments in research and clinical relevance.

Houle et al. [13] indicated that phenotypic characteristics like health, disease and evolutionary fitness have variations due to the complex interactions between genotype and environment. The authors suggested that genotype to phenotype association would be "inaccessible" without accurate phenotypic analysis such as the acquisition of crown dimensions using a $2 \mathrm{D}$ image analysis system. The genotype-phenotype map discussed was a metaphor for the multifactorial development of phenotypes due to the genetic, epigenetic and environmental influences. In essence, they were discussing the genotype-phenotype map in the context of the CAS in health and disease [13].

Dental development is a complex network within which multiple factors, including the intrauterine male hormones, influence the endocrine system and the epigenetic mechanisms controlling gene expression and development. A CAS with multiple reciprocating interactions was proposed to explore the environmental factors interaction with body systems and epigenetic mechanisms. The prenatal sex hormones and $\mathrm{Y}$ chromosome could influence the endocrine system and immune system leading to epigenetic changes, which result in enhanced gene expression. We propose that altered gene expression from this process has led to the increase in tooth size between the OS and SS females for the mesiodistal dimension.

\section{CONCLUSION}

The aim was fulfilled by measuring the MD dimension within the permanent dentition of OS and SS samples using an enhanced 2D analysis method to quantify the influence of intrauterine male hormones on tooth size and shape. The results further explore the relation of genotype to phenotype and the proposed network of interactions between male hormones and gene expression. The results indicated larger tooth crown dimensions in females from the OS cohort in comparison to the SS cohort, supporting the validity and value of using the human dentition to explore how genetic, epigenetic and environmental interactions influence in a biological CAS. The proposed modelling of these interactions explores the SRY gene on the Y chromosome influencing the development of male hormones, which transmits to the female in utero influencing her endocrine system, leading to epigenetic changes and so affecting gene expression. This demonstrates an environmental agent influencing multiple interactions in a developmental CAS. The difference in tooth size between OS and SS females provides further information of the pattern of sexual dimorphism, specifically the direct effects of the Y chromosome in males and supports the Twin Testosterone Transfer hypothesis. Further research is exploring more linear dimensions, the perimeter and surface area of the permanent teeth in the twin cohorts to investigate the dimensions most influenced by the $\mathrm{Y}$ chromosome and intrauterine male hormones.

\section{ACKNOWLEDGEMENTS}

The support of the Australian Dental Research Foundation to the first author in the form of the Undergraduate Research Grant is gratefully acknowledged.

\section{REFERENCES}

[1] Brook, A.H., The dentition: the outcomes of morphogenesis leading to variations of tooth number, size and shape. Australian Dental Journal, 59, pp. 131-142, 2014.

http://dx.doi.org/10.1111/adj.12160 
[2] Brook, A., Brook O’Donnell, M., Hone, A., Hart, E., Hughes, T., Smith R. \& Townsend, G., General and craniofacial development are complex adaptive processes influenced by diversity. Australian Dental Journal, 59S, pp. 13-22, 2014. http://dx.doi.org/10.1111/adj.12158

[3] Ribeiro, D.C., Brook, A.H., Hughes, T.E., Sampson, W.J. \& Townsend, G.C., Intrauterine hormone effects on tooth dimensions. Journal of Dental Research, 92, pp. 425-431, 2013. http://dx.doi.org/10.1177/0022034513484934

[4] Gilbert, S.F., Chromosomal Sex Determination in Mammals. Developmental Biology, 6th edn., Sinauer Associates: Sunderland, MA, 2001.

[5] Miller, E.M., Prenatal sex hormone transfer: a reason to study opposite sex twins. Personality and Individual Differences, 17, pp. 511-529, 1994. http://dx.doi.org/10.1016/0191-8869(94)90088-4

[6] Townsend, G. \& Brook, A., Genetic, epigenetic and environmental influences on dental development. Ortho Tribune, 3, pp. 4-6, 2008.

[7] Barros, S.P. \& Offenbacher, S., Epigenetics: connecting environment and genotype to phenotype and disease. Journal of Dental Research, 88, pp. 400-408, 2009. http://dx.doi.org/10.1177/0022034509335868

[8] Brook, A., Multilevel complex interactions between genetic, epigenetic and environmental factors in the aetiology of anomalies of dental development. Archives of Oral Biology, 54S, pp. 3-17, 2009.

http://dx.doi.org/10.1016/j.archoralbio.2009.09.005

[9] Townsend, G., Hughes, T., Bockmann, M., Smith, R. \& Brook, A., How studies of twins can inform our understanding of dental morphology. Frontiers of Oral Biology, 13, pp. 136-141, 2009. http://dx.doi.org/10.1159/000242406

[10] Bell, J.T. \& Spector, T.D., A twin approach to unraveling epigenetics. Trends in Genetics, 27, pp. 116-125, 2011. http://dx.doi.org/10.1016/j.tig.2010.12.005

[11] Bernal, J.E., Duran, C. \& Papiha, S.S., Transcriptional and epigenetic regulation of human microRNAs. Cancer Letters, 331(1), pp. 1-10, 2012.

[12] Yong, R., Ranjitkar, S., Townsend, G.C., Smith, R.N., Evans, A.R., Hughes, T.E., Lekkas, D. \& Brook, A.H., Dental phenomics: advancing genotype to phenotype correlations in craniofacial research. Australian Dental Journal, 59, pp. 34-47, 2014. http://dx.doi.org/10.1111/adj.12156

[13] Houle, D., Govindaraju, D.R. \& Omholt, S., Phenomics: the next challenge. Nature Reviews Genetics, 11, pp. 855-866, 2010.

http://dx.doi.org/10.1038/nrg2897 